\title{
Poly-Cauchy and Peters mixed-type polynomials
}

Dae San Kim ${ }^{1}$ and Taekyun Kim²

*Correspondence: tkkim@kw.ac.kr
${ }^{2}$ Department of Mathematics,
Kwangwoon University, Seoul,
$139-701$, Republic of Korea
Full list of author information is
available at the end of the article

available at the end of the article

\section{Abstract}

The Peters polynomials are a generalization of Boole polynomials. In this paper, we consider Peters and poly-Cauchy mixed-type polynomials and investigate the properties of those polynomials which are derived from umbral calculus. Finally, we give various identities of those polynomials associated with special polynomials.

\section{Introduction}

The Peters polynomials are defined by the generating function to be

$$
\sum_{n=0}^{\infty} S_{n}(x ; \lambda, \mu) \frac{t^{n}}{n !}=\left(1+(1+t)^{\lambda}\right)^{-\mu}(1+t)^{x} \quad(\text { see }[1]) .
$$

The first few of them are given by

$$
S_{0}(x ; \lambda, \mu)=2^{-\mu}, \quad S_{1}(x ; \lambda, \mu)=2^{-(\mu+1)}(2 x-\lambda \mu),
$$

If $\mu=1$, then $S_{n}(x ; \lambda)=S_{n}(x: \lambda, 1)$ are called Boole polynomials.

In particular, for $\mu=1, \lambda=1, S_{n}(x ; 1,1)=\mathrm{Ch}_{n}(x)$ are Changhee polynomials which are defined by

$$
\sum_{n=0}^{\infty} \mathrm{Ch}_{n}(x) \frac{t^{n}}{n !}=\frac{1}{t+2}(1+t)^{x} \quad(\text { see }[2])
$$

The generating functions for the poly-Cauchy polynomials of the first kind $C_{n}^{(k)}(x)$ and of the second kind $\hat{C}_{n}^{(k)}(x)$ are, respectively, given by

$$
\operatorname{Lif}_{k}(\log (1+t))(1+t)^{-x}=\sum_{n=0}^{\infty} C_{n}^{(k)}(x) \frac{t^{n}}{n !}
$$

and

$$
\operatorname{Lif}_{k}(-\log (1+t))(1+t)^{x}=\sum_{n=0}^{\infty} \hat{C}_{n}^{(k)}(x) \frac{t^{n}}{n !}
$$

where $\operatorname{Lif}_{k}(t)=\sum_{n=0}^{\infty} \frac{t^{n}}{n !(n+1)^{k}}(k \in \mathbb{Z})($ see $[3,4])$.

\section{黛 Springer}

○2014 Kim and Kim; licensee Springer. This is an Open Access article distributed under the terms of the Creative Commons Attribution License (http://creativecommons.org/licenses/by/2.0), which permits unrestricted use, distribution, and reproduction in any medium, provided the original work is properly cited. 
In this paper, we consider the poly-Cauchy of the first kind and Peters (respectively the poly-Cauchy of the second kind and Peters) mixed-type polynomials as follows:

$$
\left(1+(1+t)^{\lambda}\right)^{-\mu} \operatorname{Lif}_{k}(\log (1+t))(1+t)^{-x}=\sum_{n=0}^{\infty} C P_{n}^{(k)}(x ; \lambda, \mu) \frac{t^{n}}{n !}
$$

and

$$
\left(1+(1+t)^{\lambda}\right)^{-\mu} \operatorname{Lif}_{k}(-\log (1+t))(1+t)^{x}=\sum_{n=0}^{\infty} \hat{C} P_{n}^{(k)}(x ; \lambda, \mu) \frac{t^{n}}{n !}
$$

For $\alpha \in \mathbb{Z}_{\geq 0}$, the Bernoulli polynomials of order $\alpha$ are defined by the generating function to be

$$
\left(\frac{t}{e^{t}-1}\right)^{\alpha} e^{x t}=\sum_{n=0}^{\infty} B_{n}^{(\alpha)}(x) \frac{t^{n}}{n !} \quad(\text { see }[2,5-11]) .
$$

As is well known, the Frobenius-Euler polynomials of order $\alpha$ are given by

$$
\left(\frac{1-\lambda}{e^{t}-\lambda}\right)^{\alpha} e^{x t}=\sum_{n=0}^{\infty} H_{n}^{(\alpha)}(x \mid \lambda) \frac{t^{n}}{n !} \quad(\text { see }[2-14])
$$

where $\lambda \in \mathbb{C}$ with $\lambda \neq 1$ and $\alpha \in \mathbb{Z}_{\geq 0}$.

When $x=0, C P_{n}^{(k)}(0 ; \lambda, \mu)$ (or $\left.\hat{C} P_{n}^{(k)}(0 ; \lambda, \mu)\right)$ are called the poly-Cauchy of the first kind and Peters (or the poly-Cauchy of the second kind and Peters) mixed-type numbers.

The higher-order Cauchy polynomials of the first kind are defined by the generating function to be

$$
\left(\frac{t}{\log (1+t)}\right)^{\alpha}(1+t)^{-x}=\sum_{n=0}^{\infty} \mathbb{C}_{n}^{(\alpha)}(x) \frac{t^{n}}{n !} \quad\left(\alpha \in \mathbb{Z}_{\geq 0}\right)
$$

and the higher-order Cauchy polynomials of the second kind are given by

$$
\left(\frac{t}{(1+t) \log (1+t)}\right)^{\alpha}(1+t)^{x}=\sum_{n=0}^{\infty} \hat{\mathbb{C}}_{n}^{(\alpha)}(x) \frac{t^{n}}{n !} \quad\left(\alpha \in \mathbb{Z}_{\geq 0}\right) .
$$

The Stirling number of the first kind is given by

$$
(x)_{n}=x(x-1) \cdots(x-n+1)=\sum_{l=0}^{n} S_{1}(n, l) x^{l}
$$

Thus, by (10), we get

$$
(\log (1+t))^{m}=m ! \sum_{l=m}^{\infty} S_{1}(l, m) \frac{t^{l}}{l !}, \quad m \in \mathbb{Z}_{\geq 0}(\text { see }[1]) .
$$

It is easy to show that

$$
x^{(n)}=x(x+1) \cdots(x+n-1)=(-1)^{n}(-x)_{n}=\sum_{l=0}^{n} S_{1}(n, l)(-1)^{n-l} x^{l} .
$$


Let $\mathbb{C}$ be the complex number field and let $\mathcal{F}$ be the algebra of all formal power series in the variable $t$ over $\mathbb{C}$ as follows:

$$
\mathcal{F}=\left\{f(t)=\sum_{k=0}^{\infty} a_{k} \frac{t^{k}}{k !} \mid a_{k} \in \mathbb{C}\right\}
$$

Let $\mathbb{P}=\mathbb{C}[x]$ and let $\mathbb{P}^{*}$ be the vector space of all linear functionals on $\mathbb{P} .\langle L \mid p(x)\rangle$ denotes the action of the linear functional $L$ on the polynomial $p(x)$, and we recall that the vector space operations on $\mathbb{P}^{*}$ are defined by $\langle L+M \mid p(x)\rangle=\langle L \mid p(x)\rangle+\langle M \mid p(x)\rangle,\langle c L \mid p(x)\rangle=$ $c\langle L \mid p(x)\rangle$, where $c$ is a complex constant in $\mathbb{C}$.

For $f(t) \in \mathcal{F}$, let us define the linear functional on $\mathbb{P}$ by setting

$$
\left\langle f(t) \mid x^{n}\right\rangle=a_{n} \quad(n \geq 0) .
$$

Then, by (13) and (14), we get

$$
\left\langle t^{k} \mid x^{n}\right\rangle=n ! \delta_{n, k} \quad(n, k \geq 0)(\text { see }[1,15])
$$

where $\delta_{n, k}$ is the Kronecker symbol.

Let $f_{L}(t)=\sum_{k=0}^{\infty} \frac{\left\langle L \mid x^{k}\right\rangle}{k !} t^{k}$. Then, by (14), we see that $\left\langle f_{L}(t) \mid x^{n}\right\rangle=\left\langle L \mid x^{n}\right\rangle$. The map $L \longmapsto$ $f_{L}(t)$ is a vector space isomorphism from $\mathbb{P}^{*}$ onto $\mathcal{F}$. Henceforth, $\mathcal{F}$ denotes both the algebra of formal power series in $t$ and the vector space of all linear functionals on $\mathbb{P}$, and so an element $f(t)$ of $\mathcal{F}$ will be thought of as both a formal power series and a linear functional. We call $\mathcal{F}$ the umbral algebra, and the umbral calculus is the study of umbral algebra. The order $O(f)$ of the power series $f(t)(\neq 0)$ is the smallest integer for which the coefficient of $t^{k}$ does not vanish. If $O(f(t))=1$, then $f(t)$ is called a delta series; if $O(f(t))=0$, then $f(t)$ is called an invertible series. For $f(t), g(t) \in \mathcal{F}$ with $O(f(t))=1$ and $O(g(t))=0$, there exists a unique sequence $s_{n}(x)$ of polynomials such that $\left\langle g(t) f(t)^{k} \mid s_{n}(x)\right\rangle=n ! \delta_{n, k}(n, k \geq 0)$.

The sequence $s_{n}(x)$ is called the Sheffer sequence for $(g(t), f(t))$ which is denoted by $s_{n}(x) \sim(g(t), f(t))$.

For $f(t), g(t) \in \mathcal{F}$ and $p(x) \in \mathbb{P}$, we have

$$
\langle f(t) g(t) \mid p(x)\rangle=\langle f(t) \mid g(t) p(x)\rangle=\langle g(t) \mid f(t) p(x)\rangle
$$

and

$$
f(t)=\sum_{k=0}^{\infty}\left\langle f(t) \mid x^{k}\right\rangle \frac{t^{k}}{k !}, \quad p(x)=\sum_{k=0}^{\infty}\left\langle t^{k}|p(x)| \frac{x^{k}}{k !} .\right.
$$

By (16), we get

$$
p^{(k)}(0)=\left\langle t^{k} \mid p(x)\right\rangle=\left\langle 1 \mid p^{(k)}(x)\right\rangle \quad(k \geq 0),
$$

where $p^{(k)}(0)=\left.\frac{d^{k} p(x)}{d x^{k}}\right|_{x=0}$.

Thus, by (17), we have

$$
t^{k} p(x)=p^{(k)}(x)=\frac{d^{k} p(x)}{d x^{k}} \quad(\text { see }[1-3]) .
$$


Let $s_{n}(x) \sim(g(t), f(t))$. Then the following equations are known in [1]:

$$
\frac{1}{g(\bar{f}(t))} e^{x \bar{f}(t)}=\sum_{n=0}^{\infty} s_{n}(x) \frac{t^{n}}{n !} \quad \text { for all } x \in \mathbb{C},
$$

where $\bar{f}(t)$ is the compositional inverse for $f(t)$ with $f(\bar{f}(t))=t$,

$$
\begin{aligned}
& s_{n}(x)=\sum_{j=0}^{n} \frac{1}{j !}\left\langle\frac{(\bar{f}(t))^{j}}{g(\bar{f}(t))} \mid x^{n}\right\rangle x^{j}, \\
& s_{n}(x+y)=\sum_{j=0}^{n}\left(\begin{array}{l}
n \\
j
\end{array}\right) s_{j}(x) P_{n-j}(y), \quad \text { where } P_{n}(x)=g(t) s_{n}(x),
\end{aligned}
$$

and

$$
s_{n+1}(x)=\left(x-\frac{g^{\prime}(t)}{g(t)}\right) \frac{1}{f^{\prime}(t)} s_{n}(x), \quad f(t) s_{n}(x)=n s_{n-1}(x)(n \geq 0),
$$

and

$$
\frac{d}{d x} s_{n}(x)=\sum_{l=0}^{n-1}\left(\begin{array}{l}
n \\
l
\end{array}\right)\left\langle\bar{f}(t) \mid x^{n-l}\right\rangle s_{l}(x) .
$$

As is well known, the transfer formula for $p_{n}(x) \sim(1, f(t)), q_{n}(x) \sim(1, g(t))(n \geq 1)$ is given by

$$
q_{n}(x)=x\left(\frac{f(t)}{g(t)}\right)^{n} x^{-1} p_{n}(x) .
$$

$$
\begin{aligned}
\text { For } s_{n}(x) & \sim(g(t), f(t)), r_{n}(x) \sim(h(t), l(t)), \text { let } \\
s_{n}(x) & =\sum_{m=0}^{\infty} C_{n, m} r_{n}(x),
\end{aligned}
$$

where

$$
C_{n, m}=\frac{1}{m !}\left\langle\frac{h(\bar{f}(t))}{g(\bar{f}(t))}(l(\bar{f}(t)))^{m} \mid x^{n}\right\rangle \quad(\text { see }[1]) .
$$

It is known that

$$
\langle f(t) \mid x p(x)\rangle=\left\langle\partial_{t} f(t) \mid p(x)\right\rangle, \quad e^{y t} p(x)=p(x+y),
$$

where $f(t) \in \mathcal{F}$ and $p(x) \in \mathbb{P}$ (see [1-3]).

In this paper, we consider Peters and poly-Cauchy mixed-type polynomials with umbral calculus viewpoint and investigate the properties of those polynomials which are derived from umbral calculus. Finally, we give some interesting identities of those polynomials associated with special polynomials. 


\section{Poly-Cauchy and Peters mixed-type polynomials}

From (2), (3), and (19), we note that

$$
C P_{n}^{(k)}(x ; \lambda, \mu) \sim\left(\left(1+e^{-\lambda t}\right)^{\mu} \frac{1}{\operatorname{Lif}_{k}(-t)}, e^{-t}-1\right)
$$

and

$$
\hat{C} P_{n}^{(k)}(x ; \lambda, \mu) \sim\left(\left(1+e^{\lambda t}\right)^{\mu} \frac{1}{\operatorname{Lif}_{k}(-t)}, e^{t}-1\right)
$$

It is not difficult to show that

$$
\begin{aligned}
\left(1+e^{-\lambda t}\right)^{\mu} & =2^{\mu}\left(1+\frac{1}{2} \sum_{j=1}^{\infty} \frac{(-\lambda t)^{j}}{j !}\right)^{\mu} \\
& =\sum_{i=0}^{\infty} \sum_{j=0}^{\infty} \sum_{j_{1}+\cdots+j_{i}=j} 2^{\mu-i}\left(\begin{array}{c}
\mu \\
i
\end{array}\right)\left(\begin{array}{c}
j+i \\
j_{1}+1, \ldots, j_{i}+1
\end{array}\right) \frac{(-\lambda t)^{j+i}}{(j+i) !}
\end{aligned}
$$

and

$$
\begin{aligned}
\left(1+(1+t)^{\lambda}\right)^{-\mu} & =2^{-\mu}\left(1+\frac{1}{2} \sum_{j=0}^{\infty}\left(\begin{array}{c}
\lambda \\
j+1
\end{array}\right) t^{j+1}\right)^{-\mu} \\
& =\sum_{i=0}^{\infty} \sum_{j=0}^{\infty} \sum_{j_{1}+\cdots+j_{i}=j} 2^{-(\mu+i)}\left(\begin{array}{c}
-\mu \\
i
\end{array}\right)\left(\begin{array}{c}
\lambda \\
j_{1}+1
\end{array}\right) \cdots\left(\begin{array}{c}
\lambda \\
j_{i}+1
\end{array}\right) t^{j+i} .
\end{aligned}
$$

From (14), we have

$$
\begin{aligned}
C P_{n}^{(k)}(y ; \lambda, \mu) & \\
& =\left\langle\sum_{l=0}^{\infty} C P_{l}^{(k)}(y ; \lambda, \mu) \frac{t^{l}}{l !} \mid x^{n}\right\rangle \\
& =\left\langle\left(1+(1+t)^{\lambda}\right)^{-\mu} \operatorname{Lif}_{k}(\log (1+t))(1+t)^{-y} \mid x^{n}\right\rangle \\
& =\left\langle\left(1+(1+t)^{\lambda}\right)^{-\mu} \mid \sum_{l=0}^{n}\left(\begin{array}{l}
n \\
l
\end{array}\right) C_{l}^{(k)}(y) x^{n-l}\right\rangle \\
& =\sum_{l=0}^{n}\left(\begin{array}{l}
n \\
l
\end{array}\right) C_{l}^{(k)}(y)\left\langle\sum_{m=0}^{\infty} S_{m}(0 ; \lambda, \mu) \frac{t^{m}}{m !} \mid x^{n-l}\right\rangle \\
& =\sum_{l=0}^{n}\left(\begin{array}{l}
n \\
l
\end{array}\right) S_{n-l}(0 ; \lambda, \mu) C_{l}^{(k)}(y) .
\end{aligned}
$$

Therefore, by (32), we obtain the following theorem.

Theorem 1 For $n \geq 0$, we have

$$
C P_{n}^{(k)}(x ; \lambda, \mu)=\sum_{l=0}^{n}\left(\begin{array}{l}
n \\
l
\end{array}\right) S_{n-l}(0 ; \lambda, \mu) C_{l}^{(k)}(x) .
$$


Alternatively,

$$
\begin{aligned}
C P_{n}^{(k)}(y ; \lambda, \mu) & =\left\langle\sum_{l=0}^{\infty} C P_{l}^{(k)}(y ; \lambda, \mu) \frac{t^{l}}{l !} \mid x^{n}\right\rangle \\
& =\left\langle\operatorname{Lif}_{k}(\log (1+t)) \mid\left(1+(1+t)^{\lambda}\right)^{-\mu}(1+t)^{-y} x^{n}\right\rangle \\
& =\left\langle\operatorname{Lif}_{k}(\log (1+t)) \mid \sum_{l=0}^{n}\left(\begin{array}{l}
n \\
l
\end{array}\right) S_{l}(-y ; \lambda, \mu) x^{n-l}\right\rangle \\
& =\sum_{l=0}^{n}\left(\begin{array}{l}
n \\
l
\end{array}\right) S_{l}(-y ; \lambda, \mu)\left\langle\operatorname{Lif}_{k}(\log (1+t)) \mid x^{n-l}\right\rangle \\
& =\sum_{l=0}^{n}\left(\begin{array}{l}
n \\
l
\end{array}\right) S_{l}(-y ; \lambda, \mu) C_{n-l}^{(k)}(0) .
\end{aligned}
$$

Therefore, by (33), we obtain the following theorem.

Theorem 2 For $n \geq 0$, let $C_{n-l}^{(k)}(0)=C_{n-l}^{(k)}$. Then we have

$$
C P_{n}^{(k)}(x ; \lambda, \mu)=\sum_{l=0}^{n}\left(\begin{array}{l}
n \\
l
\end{array}\right) C_{n-l}^{(k)} S_{l}(-x ; \lambda, \mu) .
$$

Remark By the same method, we get

$$
\hat{C} P_{n}^{(k)}(x ; \lambda, \mu)=\sum_{l=0}^{n}\left(\begin{array}{l}
n \\
l
\end{array}\right) S_{n-l}(0 ; \lambda, \mu) \hat{C}_{l}^{(k)}(x)
$$

and

$$
\hat{C} P_{n}^{(k)}(x ; \lambda, \mu)=\sum_{l=0}^{n}\left(\begin{array}{l}
n \\
l
\end{array}\right) \hat{C}_{n-l}^{(k)} S_{l}(x ; \lambda, \mu) .
$$

From (20) and (28), we have

$$
\begin{aligned}
& C P_{n}^{(k)}(x ; \lambda, \mu) \\
& \quad=\sum_{j=0}^{n} \frac{1}{j !}\left\{\left(1+(1+t)^{\lambda}\right)^{-\mu} \operatorname{Lif}_{k}(\log (1+t))(-\log (1+t))^{j} \mid x^{n}\right) x^{j} .
\end{aligned}
$$

From (31), we note that

$$
\begin{aligned}
\langle(1+ & \left.(1+t)^{\lambda}\right)^{-\mu} \operatorname{Lif}_{k}(\log (1+t))(-\log (1+t))^{j}\left|x^{n}\right\rangle \\
= & \sum_{m=0}^{n-j} \frac{(-1)^{j}}{m !(m+1)^{k}} \sum_{l=0}^{n-j-m} \frac{(m+j) !}{(l+m+j) !} S_{1}(l+m+j, m+j) \\
& \times(n)_{l+m+j}\left\langle\left(1+(1+t)^{\lambda}\right)^{-\mu} \mid x^{n-l-m-j}\right\rangle \\
= & \sum_{m=0}^{n-j} \frac{(-1)^{j}}{m !(m+1)^{k}} \sum_{l=0}^{n-j-m} \frac{(m+j) !}{(l+m+j) !}
\end{aligned}
$$




$$
\begin{aligned}
& \times S_{1}(l+m+j, m+j)(n)_{l+m+j} \sum_{i=0}^{n-j-m-l} \sum_{r=0}^{\infty} \sum_{r_{1}+\cdots+r_{i}=r} 2^{-(\mu+i)} \\
& \times\left(\begin{array}{c}
-\mu \\
i
\end{array}\right)\left(\begin{array}{c}
\lambda \\
r_{1}+1
\end{array}\right) \cdots\left(\begin{array}{c}
\lambda \\
r_{i}+1
\end{array}\right)\left\langle t^{r+i} \mid x^{n-l-m-j}\right\rangle \\
& =2^{-\mu} n ! \sum_{m=0}^{n-j} \sum_{l=0}^{n-j-m} \sum_{i=0}^{n-j-m-l} \sum_{r_{1}+\cdots+r_{i}=n-j-m-l-i} \frac{2^{-i}(-1)^{j}(m+j) !}{m !(m+1)^{k}(l+m+j) !} \\
& \times\left(\begin{array}{c}
-\mu \\
i
\end{array}\right)\left(\begin{array}{c}
\lambda \\
r_{1}+1
\end{array}\right) \cdots\left(\begin{array}{c}
\lambda \\
r_{i}+1
\end{array}\right) S_{1}(l+m+j, m+j) .
\end{aligned}
$$

Therefore, by (36) and (37), we obtain the following theorem.

Theorem 3 For $n \geq 0$, we have

$$
\begin{aligned}
& C P_{n}^{(k)}(x ; \lambda, \mu) \\
& =2^{-\mu} n ! \sum_{j=0}^{n} \frac{(-1)^{j}}{j !}\left\{\sum_{m=0}^{n-j} \sum_{l=0}^{n-j-m} \sum_{i=0}^{n-j-m-l} \sum_{r_{1}+\cdots+r_{i}=n-j-m-l-i} \frac{2^{-i}}{m !(m+1)^{k}}\right. \\
& \left.\quad \times \frac{(m+j) !}{(l+m+j) !}\left(\begin{array}{c}
-\mu \\
i
\end{array}\right)\left(\begin{array}{c}
\lambda \\
r_{1}+1
\end{array}\right) \cdots\left(\begin{array}{c}
\lambda \\
r_{i}+1
\end{array}\right) S_{1}(l+m+j, m+j)\right\} x^{j}
\end{aligned}
$$

Remark By the same method as Theorem 3, we get

$$
\begin{aligned}
\hat{C} P_{n}^{(k)}(x ; \lambda, \mu) \\
=2^{-\mu} n ! \sum_{j=0}^{n} \frac{1}{j !}\left\{\sum_{m=0}^{n-j} \sum_{l=0}^{n-j-m} \sum_{i=0}^{n-j-m-l} \sum_{r_{1}+\cdots+r_{i}=n-j-m-l-i} \frac{2^{-i}(-1)^{m}}{m !(m+1)^{k}}\right. \\
\quad \times \frac{(m+j) !}{(l+m+j) !}\left(\begin{array}{c}
-\mu \\
i
\end{array}\right) \\
\left.\quad \times\left(\begin{array}{c}
\lambda \\
r_{1}+1
\end{array}\right) \cdots\left(\begin{array}{c}
\lambda \\
r_{i}+1
\end{array}\right) S_{1}(l+m+j, m+j)\right\} x^{j} .
\end{aligned}
$$

From (28), we note that

$$
\left(1+e^{-\lambda t}\right)^{\mu} \frac{1}{\operatorname{Lif}_{k}(-t)} C P_{n}^{(k)}(x ; \lambda, \mu) \sim\left(1, e^{-t}-1\right)
$$

and

$$
x^{n} \sim(1, t)
$$

By (24), (39), and (40), we get

$$
\begin{aligned}
\left(1+e^{-\lambda t}\right)^{\mu} \frac{1}{\operatorname{Lif}_{k}(-t)} C P_{n}^{(k)}(x ; \lambda, \mu) \\
=x\left(\frac{t}{e^{-t}-1}\right)^{n} x^{n-1}
\end{aligned}
$$




$$
\begin{aligned}
& =(-1)^{n} x\left(\frac{-t}{e^{-t}-1}\right)^{n} x^{n-1} \\
& =(-1)^{n} \sum_{l=0}^{n-1}(-1)^{l} B_{l}^{(n)}\left(\begin{array}{c}
n-1 \\
l
\end{array}\right) x^{n-l} .
\end{aligned}
$$

Thus, by (41), we see that

$$
\begin{aligned}
C P_{n}^{(k)}(x ; \lambda, \mu) & (-1)^{n} \sum_{l=0}^{n-1}(-1)^{l}\left(\begin{array}{c}
n-1 \\
l
\end{array}\right) B_{l}^{(n)}\left(1+e^{-\lambda t}\right)^{-\mu} \operatorname{Lif}_{k}(-t) x^{n-l} \\
= & (-1)^{n} \sum_{l=0}^{n-1}(-1)^{l}\left(\begin{array}{c}
n-1 \\
l
\end{array}\right) B_{l}^{(n)} \sum_{m=0}^{n-l} \frac{(-1)^{m}\left(\begin{array}{c}
n-l \\
m
\end{array}\right)}{(m+1)^{k}}\left(1+e^{-\lambda t}\right)^{-\mu} x^{n-l-m} \\
= & (-1)^{n} \sum_{l=0}^{n-1}(-1)^{l}\left(\begin{array}{c}
n-1 \\
l
\end{array}\right) B_{l}^{(n)} \sum_{m=0}^{n-l} \frac{(-1)^{m}\left(\begin{array}{c}
n-l \\
m
\end{array}\right)}{(m+1)^{k}} \sum_{i=0}^{\infty} \sum_{j=0}^{\infty} \sum_{j_{1}+\cdots+j_{n}=j} 2^{-\mu-i}\left(\begin{array}{c}
-\mu \\
i
\end{array}\right) \\
& \times\left(\begin{array}{c}
j+i \\
j_{1}+1, \ldots, j_{i}+1
\end{array}\right) \frac{(-\lambda t)^{j+i}}{(j+i) !} x^{n-l-m} \sum_{l=0}^{n-l} \sum_{m=0}^{n-l-m} n-l-m-i \\
= & \left.(-1)^{n} \sum_{i=0}^{n} \sum_{j=0}^{j_{1}+\cdots+j_{i}=n-l-m-i-r} \sum^{n-1}\right)^{n-r} \frac{2^{-\mu-i} \lambda^{n-l-m-r}}{(m+1)^{k}}\left(\begin{array}{c}
n-1 \\
l
\end{array}\right) \\
& \times\left(\begin{array}{c}
n-l \\
m
\end{array}\right)\left(\begin{array}{c}
-\mu \\
i
\end{array}\right)\left(\begin{array}{c}
n-l-m-r \\
j_{1}+1, \ldots, j_{i}+1
\end{array}\right)\left(\begin{array}{c}
n-l-m \\
r
\end{array}\right) B_{l}^{(n)} x^{r} .
\end{aligned}
$$

Therefore, by (42), we obtain the following theorem.

Theorem 4 For $n \geq 0$, we have

$$
\begin{aligned}
& C P_{n}^{(k)}(x ; \lambda, \mu) \\
& =\frac{\lambda^{n}}{2^{\mu}} \sum_{r=0}^{n}\left(-\lambda^{-1}\right)^{r}\left\{\sum_{l=0}^{n-r} \sum_{m=0}^{n-r-l} \sum_{i=0}^{n-r-l-m} \sum_{j_{1}+\cdots+j_{i}=n-r-l-m-i} \frac{2^{-i} \lambda^{-l-m}}{(m+1)^{k}}\left(\begin{array}{c}
n-1 \\
l
\end{array}\right)\right. \\
& \left.\quad \times\left(\begin{array}{c}
n-l \\
m
\end{array}\right)\left(\begin{array}{c}
-\mu \\
i
\end{array}\right)\left(\begin{array}{c}
n-r-l-m \\
j_{1}+1, \ldots, j_{i}+1
\end{array}\right)\left(\begin{array}{c}
n-l-m \\
r
\end{array}\right) B_{l}^{(n)}\right\} x^{r} .
\end{aligned}
$$

Remark By the same method as Theorem 4, we get

$$
\begin{aligned}
\hat{C} P_{n}^{(k)}(x ; \lambda, \mu) & \\
= & \frac{\lambda^{n}}{2^{\mu}} \sum_{r=0}^{n} \lambda^{-r}\left\{\sum_{l=0}^{n-r} \sum_{m=0}^{n-l-r} \sum_{i=0}^{n-l-m-r} \sum_{j_{1}+\cdots+j_{i}=n-r-l-m-i} \frac{(-1)^{m} 2^{-i} \lambda^{-l-m}}{(m+1)^{k}}\left(\begin{array}{c}
n-1 \\
l
\end{array}\right)\right. \\
& \left.\times\left(\begin{array}{c}
n-l \\
m
\end{array}\right)\left(\begin{array}{c}
-\mu \\
i
\end{array}\right)\left(\begin{array}{c}
n-r-l-m \\
j_{1}+1, \ldots, j_{i}+1
\end{array}\right)\left(\begin{array}{c}
n-l-m \\
r
\end{array}\right) B_{l}^{(m)}\right\} x^{r} .
\end{aligned}
$$


From (12), we note that

$$
x^{(n)}=x(x+1) \cdots(x+n-1) \sim\left(1,1-e^{-t}\right) .
$$

Thus, by (44), we see that

$$
(-1)^{n} x^{(n)}=(-x)_{n}=\sum_{m=0}^{n} S_{1}(n, m)(-x)^{m} \sim\left(1, e^{-t}-1\right)
$$

and

$$
\left(1+e^{-\lambda t}\right)^{\mu} \frac{1}{\operatorname{Lif}_{k}(-t)} C P_{n}^{(k)}(x ; \lambda, \mu) \sim\left(1, e^{-t}-1\right)
$$

From (45) and (46), we have

$$
\begin{aligned}
(1 & \left.+e^{-\lambda t}\right)^{\mu} \frac{1}{\operatorname{Lif}_{k}(-t)} C P_{n}^{(k)}(x ; \lambda, \mu) \\
& =(-1)^{n} x^{(n)} \\
& =\sum_{l=0}^{n} S_{1}(n, l)(-x)^{l} .
\end{aligned}
$$

Thus, by (47), we get

$$
\begin{aligned}
& C P_{n}^{(k)}(x ; \lambda, \mu) \\
& =\sum_{l=0}^{n} S_{1}(n, l)(-1)^{l}\left(1+e^{-\lambda t}\right)^{-\mu} \operatorname{Lif}_{k}(-t) x^{l} \\
& =\sum_{l=0}^{n} S_{1}(n, l)(-1)^{l} \sum_{m=0}^{l} \frac{(-1)^{m}\left(\begin{array}{l}
l \\
m
\end{array}\right)}{(m+1)^{k}}\left(1+e^{-\lambda t}\right)^{-\mu} x^{l-m} \\
& =\sum_{l=0}^{n} S_{1}(n, l)(-1)^{l} \sum_{m=0}^{l} \frac{(-1)^{m}\left(\begin{array}{c}
l \\
m
\end{array}\right)}{(m+1)^{k}} \sum_{i=0}^{\infty} \sum_{j=0}^{\infty} \sum_{j_{1}+\cdots+j_{i}=j} 2^{-\mu-i} \\
& \times\left(\begin{array}{c}
-\mu \\
i
\end{array}\right)\left(\begin{array}{c}
j+i \\
j_{1}+1, \ldots, j_{i}+1
\end{array}\right) \frac{(-\lambda)^{j+i}}{(j+i) !} t^{j+i} x^{l-m} \\
& =\sum_{l=0}^{n} \sum_{m=0}^{l} \sum_{i=0}^{l-m} \sum_{r=0}^{l-m-i} \sum_{j_{1}+\cdots+j_{i}=l-m-i-r}(-1)^{r} \frac{2^{-\mu-i} \lambda^{l-m-r}}{(m+1)^{k}} \\
& \times\left(\begin{array}{c}
l \\
m
\end{array}\right)\left(\begin{array}{c}
-\mu \\
i
\end{array}\right)\left(\begin{array}{c}
l-m-r \\
j_{1}+1, \ldots, j_{i}+1
\end{array}\right)\left(\begin{array}{c}
l-m \\
r
\end{array}\right) S_{1}(n, l) x^{r} \\
& =2^{-\mu} \sum_{r=0}^{n}\left(-\lambda^{-1}\right)^{r}\left\{\sum_{l=r}^{n} \sum_{m=0}^{l-r} \sum_{i=0}^{l-r-m} \sum_{j_{1}+\cdots+j_{i}=l-r-m-i} \frac{2^{-i} \lambda^{l-m}}{(m+1)^{k}}\right. \\
& \left.\times\left(\begin{array}{c}
l \\
m
\end{array}\right)\left(\begin{array}{c}
-\mu \\
i
\end{array}\right)\left(\begin{array}{c}
l-m-r \\
j_{1}+1, \ldots, j_{i}+1
\end{array}\right)\left(\begin{array}{c}
l-m \\
r
\end{array}\right) S_{1}(n, l)\right\} x^{r} .
\end{aligned}
$$

Therefore, by (48), we obtain the following theorem. 
Theorem 5 For $n \geq 0$, we have

$$
\begin{aligned}
& C P_{n}^{(k)}(x ; \lambda, \mu) \\
& =2^{-\mu} \sum_{r=0}^{n}\left(-\lambda^{-1}\right)^{r}\left\{\sum_{l=r}^{n} \sum_{m=0}^{l-r} \sum_{i=0}^{l-r-m} \sum_{j_{1}+\cdots+j_{i}=l-r-m-i} \frac{2^{-i} \lambda^{l-m}}{(m+1)^{k}}\right. \\
& \left.\quad \times\left(\begin{array}{c}
l \\
m
\end{array}\right)\left(\begin{array}{c}
-\mu \\
i
\end{array}\right)\left(\begin{array}{c}
l-m-r \\
j_{1}+1, \ldots, j_{i}+1
\end{array}\right)\left(\begin{array}{c}
l-m \\
r
\end{array}\right) S_{1}(n, l)\right\} x^{r} .
\end{aligned}
$$

It is easy to see that

$$
\left(1+e^{\lambda t}\right)^{\mu} \frac{1}{\operatorname{Lif}_{k}(-t)} \hat{C} P_{n}^{(k)}(x ; \lambda, \mu) \sim\left(1, e^{t}-1\right)
$$

and

$$
(x)_{n}=x(x-1) \cdots(x-n+1)=\sum_{l=0}^{n} S_{1}(n, l) x^{l} \sim\left(1, e^{t}-1\right) .
$$

By the same method as Theorem 5, we get

$$
\begin{aligned}
& \hat{C} P_{n}^{(k)}(x ; \lambda, \mu) \\
& =2^{-\mu} \sum_{r=0}^{n} \lambda^{-r}\left\{\sum_{l=r}^{n} \sum_{m=0}^{l-r} \sum_{i=0}^{l-r-m} \sum_{j_{1}+\cdots+j_{i}=l-r-m-i} \frac{(-1)^{m} 2^{-i} \lambda^{l-m}}{(m+1)^{k}}\right. \\
& \left.\quad \times\left(\begin{array}{c}
l \\
m
\end{array}\right)\left(\begin{array}{c}
-\mu \\
i
\end{array}\right)\left(\begin{array}{c}
l-m-r \\
j_{1}+1, \ldots, j_{i}+1
\end{array}\right)\left(\begin{array}{c}
l-m \\
r
\end{array}\right) S_{1}(n, l)\right\} x^{r} .
\end{aligned}
$$

From (20) and (28), we have

$$
\begin{aligned}
& C P_{n}^{(k)}(x ; \lambda, \mu) \\
& \quad=\sum_{j=0}^{n} \frac{1}{j !}\left(\left(1+(1+t)^{\lambda}\right)^{-\mu} \operatorname{Lif}_{k}(\log (1+t))(-\log (1+t))^{j} \mid x^{n}\right) x^{j} .
\end{aligned}
$$

Now, we observe that

$$
\begin{aligned}
\left\langle\left(1+(1+t)^{\lambda}\right)^{-\mu} \operatorname{Lif}_{k}(\log (1+t))(-\log (1+t))^{j} \mid x^{n}\right\rangle \\
=(-1)^{j}\left\langle\log (1+t)^{j} \mid \sum_{m=0}^{\infty} C P_{m}^{(k)}(0 ; \lambda, \mu) \frac{t^{m}}{m !} x^{n}\right\rangle \\
=(-1)^{j} \sum_{m=0}^{n}\left(\begin{array}{l}
n \\
m
\end{array}\right) C P_{m}^{(k)}(0 ; \lambda, \mu)\left\langle(\log (1+t))^{j} \mid x^{n-m}\right\rangle \\
=(-1)^{j} \sum_{m=0}^{n}\left(\begin{array}{c}
n \\
m
\end{array}\right) C P_{m}^{(k)}(0 ; \lambda, \mu) j ! S_{1}(n-m, j) .
\end{aligned}
$$

Therefore, by (52) and (53), we obtain the following theorem. 
Theorem 6 For $n \geq 0$, we have

$$
C P_{n}^{(k)}(x ; \lambda, \mu)=\sum_{j=0}^{n}(-1)^{j}\left\{\sum_{m=0}^{n}\left(\begin{array}{l}
n \\
m
\end{array}\right) S_{1}(n-m, j) C P_{m}^{(k)}(0 ; \lambda, \mu)\right\} x^{j} .
$$

Remark By the same method as Theorem 6, we get

$$
\hat{C} P_{n}^{(k)}(x ; \lambda, \mu)=\sum_{j=0}^{n}\left\{\sum_{m=0}^{n}\left(\begin{array}{l}
n \\
m
\end{array}\right) S_{1}(n-m, j) \hat{C} P_{n}^{(k)}(0 ; \lambda, \mu)\right\} x^{j} .
$$

From (21), we have

$$
C P_{n}^{(k)}(x+y ; \lambda, \mu)=\sum_{j=0}^{n}(-1)^{j}\left(\begin{array}{l}
n \\
j
\end{array}\right) C P_{n-j}^{(k)}(x ; \lambda, \mu) y^{(j)}
$$

and

$$
\hat{C} P_{n}^{(k)}(x+y ; \lambda, \mu)=\sum_{j=0}^{n}\left(\begin{array}{l}
n \\
j
\end{array}\right) \hat{C} P_{n-j}^{(k)}(x ; \lambda, \mu)(y)_{j} .
$$

By (22) and (28), we get

$$
\left(e^{-t}-1\right) C P_{n}^{(k)}(x ; \lambda, \mu)=n C P_{n-1}^{(k)}(x ; \lambda, \mu)
$$

and

$$
\left(e^{-t}-1\right) C P_{n}^{(k)}(x ; \lambda, \mu)=C P_{n}^{(k)}(x-1 ; \lambda, \mu)-C P_{n}^{(k)}(x ; \lambda, \mu) .
$$

Therefore, by (57) and (58), we obtain the following theorem.

Theorem 7 For $n \geq 0$, we have

$$
C P_{n}^{(k)}(x-1 ; \lambda, \mu)-C P_{n}^{(k)}(x ; \lambda, \mu)=n C P_{n-1}^{(k)}(x ; \lambda, \mu) .
$$

Remark By the same method as Theorem 7, we get

$$
\hat{C} P_{n}^{(k)}(x+1 ; \lambda, \mu)-\hat{C} P_{n}^{(k)}(x ; \lambda, \mu)=n \hat{C} P_{n-1}^{(k)}(x ; \lambda, \mu) .
$$

From (22), (28), and (29), we have

$$
\begin{aligned}
C P_{n+1}^{(k)}(x ; 1, \mu)= & -x C P_{n}^{(k)}(x+1 ; 1, \mu)+\mu \sum_{m=0}^{n}\left(-\frac{1}{2}\right)^{m+1}(n)_{m} C P_{n-m}^{(k)}(x ; 1, \mu) \\
& +2^{-\mu} \sum_{r=0}^{n}(-1)^{r}\left\{\sum_{m=r}^{n} \sum_{l=r}^{m} \sum_{i=0}^{l-r} \sum_{j_{1}+\cdots+j_{i}=l-i-r} \frac{2^{-i}}{(m-l+2)^{k}}\left(\begin{array}{c}
m \\
l
\end{array}\right)\right. \\
& \left.\times\left(\begin{array}{c}
-\mu \\
i
\end{array}\right)\left(\begin{array}{c}
l-r \\
j_{1}+1, \ldots, j_{i}+1
\end{array}\right)\left(\begin{array}{l}
l \\
r
\end{array}\right) S_{1}(n, m)\right\}(x+1)^{r}
\end{aligned}
$$


and

$$
\begin{aligned}
\hat{C} P_{n+1}^{(k)}(x ; 1, \mu) \\
=x \hat{C} P_{n}^{(k)}(x-1 ; 1, \mu)+\mu \sum_{m=0}^{n}\left(-\frac{1}{2}\right)^{m+1}(n)_{m} \hat{C} P_{n-m}^{(k)}(x ; 1, \mu) \\
\quad-2^{-\mu} \sum_{r=0}^{n}\left\{\sum_{m=r}^{n} \sum_{l=r}^{m} \sum_{i=0}^{l-r} \sum_{j_{1}+\cdots+j_{i}=l-i-r} \frac{(-1)^{m-l} 2^{-i}}{(m-l+2)^{k}}\left(\begin{array}{c}
m \\
l
\end{array}\right)\left(\begin{array}{c}
-\mu \\
i
\end{array}\right)\right. \\
\left.\quad \times\left(\begin{array}{c}
l-r \\
j_{1}+1, \ldots, j_{i}+1
\end{array}\right)\left(\begin{array}{l}
l \\
r
\end{array}\right) S_{1}(n, m)\right\}(x-1)^{r} .
\end{aligned}
$$

By (14) and (27), we get

$$
\begin{aligned}
C P_{n}^{(k)}(y ; \lambda, \mu) & \left\langle\sum_{l=0}^{\infty} C P_{l}^{(k)}(y ; \lambda, \mu) \frac{t^{l}}{l !} \mid x^{n}\right\rangle \\
= & \left\langle\left(1+(1+t)^{\lambda}\right)^{-\mu} \operatorname{Lif}_{k}(\log (1+t))(1+t)^{-y} \mid x \cdot x^{n-1}\right\rangle \\
= & \left\langle\partial_{t}\left(\left(1+(1+t)^{\lambda}\right)^{-\mu} \operatorname{Lif}_{k}(\log (1+t))(1+t)^{-y}\right) \mid x^{n-1}\right\rangle \\
= & \left\langle\left(\partial_{t}\left(1+(1+t)^{\lambda}\right)^{-\mu}\right) \operatorname{Lif}_{k}(\log (1+t))(1+t)^{-y} \mid x^{n-1}\right\rangle \\
& +\left\langle\left(1+(1+t)^{\lambda}\right)^{-\mu}\left(\partial_{t} \operatorname{Lif}_{k}(\log (1+t))\right)(1+t)^{-y} \mid x^{n-1}\right\rangle \\
& +\left\langle\left(1+(1+t)^{\lambda}\right)^{-\mu} \operatorname{Lif}_{k}(\log (1+t))\left(\partial_{t}(1+t)^{-y}\right) \mid x^{n-1}\right\rangle \\
= & -\mu \lambda\left\langle\left(1+(1+t)^{\lambda}\right)^{-\mu-1} \operatorname{Lif}_{k}(\log (1+t))(1+t)^{-(y-\lambda+1)} \mid x^{n-1}\right\rangle \\
& -y\left\langle\left(1+(1+t)^{\lambda}\right)^{-\mu}\left(\operatorname{Lif}_{k}(\log (1+t))\right)(1+t)^{-y-1} \mid x^{n-1}\right\rangle \\
& +\left\langle\left(1+(1+t)^{\lambda}\right)^{-\mu}\left(\partial_{t} \operatorname{Lif}_{k}(\log (1+t))\right)(1+t)^{-y} \mid x^{n-1}\right\rangle \\
= & -\mu \lambda C P_{n-1}^{(k)}(y-\lambda+1 ; \lambda, \mu+1)-y C P_{n-1}^{(k)}(y+1 ; \lambda, \mu) \\
& +\left\langle\left(1+(1+t)^{\lambda}\right)^{-\mu} \frac{\operatorname{Lif}_{k-1}(\log (1+t))-\operatorname{Lif}_{k}(\log (1+t))}{(1+t) \log (1+t)}(1+t)^{-y} \mid x^{n-1}\right\rangle .
\end{aligned}
$$

Now, we observe that

$$
\begin{aligned}
\langle & \left(1+(1+t)^{\lambda}\right)^{-\mu} \frac{\operatorname{Lif}_{k-1}(\log (1+t))-\operatorname{Lif}_{k}(\log (1+t))}{(1+t) \log (1+t)}(1+t)^{-y}\left|x^{n-1}\right\rangle \\
= & \left\langle\left(1+(1+t)^{\lambda}\right)^{-\mu} \frac{\operatorname{Lif}_{k-1}(\log (1+t))-\operatorname{Lif}_{k}(\log (1+t))}{t}(1+t)^{-y}\right| \\
& \left.\frac{t}{(1+t) \log (1+t)} x^{n-1}\right\rangle \\
= & \sum_{l=0}^{n-1}\left(\begin{array}{c}
n-1 \\
l
\end{array}\right) \hat{\mathbb{C}}_{n-1-l}^{(1)}(0) \\
& \times\left\langle\left(1+(1+t)^{\lambda}\right)^{-\mu} \frac{\operatorname{Lif}_{k-1}(\log (1+t))-\operatorname{Lif}_{k}(\log (1+t))}{t}(1+t)^{-y} \mid x^{l}\right\rangle
\end{aligned}
$$




$$
\begin{aligned}
= & \sum_{l=0}^{n-1}\left(\begin{array}{c}
n-1 \\
l
\end{array}\right) \hat{\mathbb{C}}_{n-1-l}^{(1)}(0) \\
& \times\left\langle\left(1+(1+t)^{\lambda}\right)^{-\mu} \frac{\operatorname{Lif}_{k-1}(\log (1+t))-\operatorname{Lif}_{k}(\log (1+t))}{t}(1+t)^{-y} \mid t \frac{x^{l+1}}{l+1}\right\rangle \\
= & \frac{1}{n} \sum_{l=0}^{n-1}\left(\begin{array}{c}
n \\
l+1
\end{array}\right) \hat{\mathbb{C}}_{n-1-l}^{(1)}(0)\left\{C P_{l+1}^{(k-1)}(y ; \lambda, \mu)-C P_{l+1}^{(k)}(y ; \lambda, \mu)\right\} .
\end{aligned}
$$

Therefore, by (62) and (63), we obtain the following theorem.

Theorem 8 For $n \geq 0$, we have

$$
\begin{aligned}
& C P_{n}^{(k)}(x ; \lambda, \mu) \\
& =-\mu \lambda C P_{n-1}^{(k)}(x-\lambda+1 ; \lambda, \mu+1)-x C P_{n-1}^{(k)}(x+1 ; \lambda, \mu) \\
& \quad+\frac{1}{n} \sum_{l=0}^{n-1}\left(\begin{array}{c}
n \\
l+1
\end{array}\right) \hat{C}_{n-1-l}\left\{C P_{l+1}^{(k-1)}(x ; \lambda, \mu)-C P_{l+1}^{(k)}(x ; \lambda, \mu)\right\},
\end{aligned}
$$

where $\hat{C}_{n-1-l}=\hat{\mathbb{C}}_{n-1-l}^{(1)}(0)$.

Remark By the same method as Theorem 8, we get

$$
\begin{aligned}
\hat{C} P_{n}^{(k)}(x ; \lambda, \mu) \\
=-\mu \lambda \hat{C} P_{n-1}^{(k)}(x+\lambda-1 ; \lambda, \mu+1)+x \hat{C} P_{n-1}^{(k)}(x-1 ; \lambda, \mu) \\
\quad+\frac{1}{n} \sum_{l=0}^{n-1}\left(\begin{array}{c}
n \\
l+1
\end{array}\right) \hat{C}_{n-1-l}\left(\hat{C} P_{l+1}^{(k-1)}(x ; \lambda, \mu)-\hat{C} P_{l+1}^{(k)}(x ; \lambda, \mu)\right) .
\end{aligned}
$$

By (23), we get

$$
\begin{aligned}
\frac{d}{d x} & C P_{n}^{(k)}(x ; \lambda, \mu) \\
& =\sum_{l=0}^{n-1}\left(\begin{array}{l}
n \\
l
\end{array}\right)\left\langle-\log (1+t) \mid x^{n-l}\right\rangle C P_{l}^{(k)}(x ; \lambda, \mu) \\
& =\sum_{l=0}^{n-1}\left(\begin{array}{l}
n \\
l
\end{array}\right)\left\langle\sum_{m=1}^{\infty} \frac{(-1)^{m}}{m} t^{m} \mid x^{n-l}\right\rangle C P_{l}^{(k)}(x ; \lambda, \mu) \\
& =\sum_{l=0}^{n-1}\left(\begin{array}{l}
n \\
l
\end{array}\right) \sum_{m=1}^{\infty} \frac{(-1)^{m}}{m}\left\langle t^{m} \mid x^{n-l}\right\rangle C P_{l}^{(k)}(x ; \lambda, \mu) \\
& =\sum_{l=0}^{n-1}\left(\begin{array}{l}
n \\
l
\end{array}\right)(-1)^{n-l} C P_{l}^{(k)}(x ; \lambda, \mu)(n-l-1) ! \\
& =n ! \sum_{l=0}^{n-1} \frac{(-1)^{n-l}}{(n-l) l !} C P_{l}^{(k)}(x ; \lambda, \mu) .
\end{aligned}
$$


By the same method as (65), we get

$$
\begin{aligned}
& \frac{d}{d x} \hat{C} P_{n}^{(k)}(x ; \lambda, \mu) \\
& \quad=n ! \sum_{l=0}^{n-1} \frac{(-1)^{n-l-1}}{(n-l) l !} \hat{C} P_{l}^{(k)}(x ; \lambda, \mu) .
\end{aligned}
$$

Now, we compute the following equation in two different ways:

$$
\left\langle\left(1+(1+t)^{\lambda}\right)^{-\mu} \operatorname{Lif}_{k}(-\log (1+t))(\log (1+t))^{m} \mid x^{n}\right\rangle .
$$

On the one hand,

$$
\begin{aligned}
\langle(1+ & \left.(1+t)^{\lambda}\right)^{-\mu} \operatorname{Lif}_{k}(-\log (1+t))(\log (1+t))^{m}\left|x^{n}\right\rangle \\
& =\left\langle\left(1+(1+t)^{\lambda}\right)^{-\mu} \operatorname{Lif}_{k}(-\log (1+t)) \mid(\log (1+t))^{m} x^{n}\right\rangle \\
& =\sum_{l=0}^{n-m} m !\left(\begin{array}{c}
n \\
l+m
\end{array}\right) S_{1}(l+m, m)\left\langle\left(1+(1+t)^{\lambda}\right)^{-\mu} \operatorname{Lif}_{k}(-\log (1+t)) \mid x^{n-l-m}\right\rangle \\
& =\sum_{l=0}^{n-m} m !\left(\begin{array}{c}
n \\
l
\end{array}\right) S_{1}(n-l, m) \hat{C} P_{l}^{(k)}(0 ; \lambda, \mu) .
\end{aligned}
$$

On the other hand,

$$
\begin{aligned}
\langle(1+ & \left.(1+t)^{\lambda}\right)^{-\mu} \operatorname{Lif}_{k}(-\log (1+t))(\log (1+t))^{m}\left|x^{n}\right\rangle \\
= & \left\langle\partial_{t}\left(\left(1+(1+t)^{\lambda}\right)^{-\mu} \operatorname{Lif}_{k}(-\log (1+t))(\log (1+t))^{m}\right) \mid x^{n-1}\right\rangle \\
= & \left\langle\left(\partial_{t}\left(1+(1+t)^{\lambda}\right)^{-\mu}\right) \operatorname{Lif}_{k}(-\log (1+t))(\log (1+t))^{m} \mid x^{n-1}\right\rangle \\
& +\left\langle\left(1+(1+t)^{\lambda}\right)^{-\mu}\left(\partial_{t} \operatorname{Lif}_{k}(-\log (1+t))\right)(\log (1+t))^{m} \mid x^{n-1}\right\rangle \\
& +\left\langle\left(1+(1+t)^{\lambda}\right)^{-\mu} \operatorname{Lif}_{k}(-\log (1+t))\left(\partial_{t}(\log (1+t))^{m}\right) \mid x^{n-1}\right\rangle .
\end{aligned}
$$

Therefore, by (67) and (68), we obtain the following theorem.

Theorem 9 For $n \in \mathbb{N}$ with $n \geq 2$, let $n-1 \geq m \geq 1$. Then we have

$$
\begin{aligned}
& m \sum_{l=0}^{n-m}\left(\begin{array}{l}
n \\
l
\end{array}\right) S_{1}(n-l, m) \hat{C} P_{l}^{(k)}(0 ; \lambda, \mu) \\
& =-\mu \lambda m \sum_{l=0}^{n-1-m}\left(\begin{array}{c}
n-1 \\
l
\end{array}\right) S_{1}(n-1-l, m) \hat{C} P_{l}^{(k)}(\lambda-1 ; \lambda, \mu+1) \\
& \quad+\sum_{l=0}^{n-m}\left(\begin{array}{c}
n-1 \\
l
\end{array}\right) S_{1}(n-1-l, m-1) \hat{C} P_{l}^{(k-1)}(-1 ; \lambda, \mu) \\
& \quad+(m-1) \sum_{l=0}^{n-m}\left(\begin{array}{c}
n-1 \\
l
\end{array}\right) S_{1}(n-1-l, m-1) \hat{C} P_{l}^{(k)}(-1 ; \lambda, \mu) .
\end{aligned}
$$


Remark By the same method as Theorem 9, we get

$$
\begin{aligned}
& m \sum_{l=0}^{n-m}\left(\begin{array}{l}
n \\
l
\end{array}\right) S_{1}(n-l, m) C P_{l}^{(k)}(0 ; \lambda, \mu) \\
& =-\mu \lambda m \sum_{l=0}^{n-1-m}\left(\begin{array}{c}
n-1 \\
l
\end{array}\right) S_{1}(n-1-l, m) C P_{l}^{(k)}(1-\lambda ; \lambda, \mu+1) \\
& \quad+\sum_{l=0}^{n-m}\left(\begin{array}{c}
n-1 \\
l
\end{array}\right) S_{1}(n-1-l, m-1) C P_{l}^{(k-1)}(1 ; \lambda, \mu) \\
& \quad+(m-1) \sum_{l=0}^{n-m}\left(\begin{array}{c}
n-1 \\
l
\end{array}\right) S_{1}(n-1-l, m-1) C P_{l}^{(k)}(-1 ; \lambda, \mu)
\end{aligned}
$$

where $n-1 \geq m \geq 1$.

Let us consider the following two Sheffer sequences:

$$
C P_{n}^{(k)}(x ; \lambda, \mu) \sim\left(\left(1+e^{-\lambda t}\right)^{\mu} \frac{1}{\operatorname{Lif}_{k}(-t)}, e^{-t}-1\right)
$$

and

$$
B_{n}^{(s)}(x) \sim\left(\left(\frac{e^{t}-1}{t}\right)^{s}, t\right) \quad\left(s \in \mathbb{Z}_{\geq 0}\right)
$$

Let

$$
C P_{n}^{(k)}(x ; \lambda, \mu)=\sum_{m=0}^{n} C_{n, m} B_{m}^{(s)}(x) .
$$

Then, by (26), we get

$$
\begin{aligned}
& C_{n, m}=\frac{1}{m !}\left\langle\frac{\left(\frac{e^{-\log (1+t)}-1}{-\log (1+t)}\right)^{s}}{\left(1+e^{\lambda \log (1+t)}\right)^{\mu}} \operatorname{Lif}_{k}(\log (1+t))(-\log (1+t))^{m} \mid x^{n}\right\rangle \\
& =\frac{(-1)^{m}}{m !}\left\langle\left(1+(1+t)^{\lambda}\right)^{-\mu} \operatorname{Lif}_{k}(\log (1+t))\right. \\
& \times(1+t)^{-s}\left(\frac{t}{\log (1+t)}\right)^{s}\left|(\log (1+t))^{m} x^{n}\right\rangle \\
& =\frac{(-1)^{m}}{m !} \sum_{l=0}^{n-m} m !\left(\begin{array}{c}
n \\
l+m
\end{array}\right) S_{1}(l+m, m) \sum_{i=0}^{n-l-m}\left(\begin{array}{c}
n-l-m \\
i
\end{array}\right) \mathbb{C}_{i}^{(s)} \\
& \times\left\langle\left(1+(1+t)^{\lambda}\right)^{-\mu} \operatorname{Lif}_{k}(\log (1+t))(1+t)^{-s} \mid x^{n-l-m-i}\right\rangle \\
& =(-1)^{m} \sum_{l=0}^{n-m}\left(\begin{array}{l}
n \\
l
\end{array}\right) S_{1}(n-l, m) \sum_{i=0}^{l}\left(\begin{array}{l}
l \\
i
\end{array}\right) \mathbb{C}_{i}^{(s)} C P_{l-i}^{(k)}(s ; \lambda, \mu) .
\end{aligned}
$$

Therefore, by (71) and (72), we obtain the following theorem. 
Theorem 10 For $n \geq 0$, we have

$$
C P_{n}^{(k)}(x ; \lambda, \mu)=\sum_{m=0}^{n}(-1)^{m}\left\{\sum_{l=0}^{n-m} \sum_{i=0}^{l}\left(\begin{array}{l}
n \\
l
\end{array}\right)\left(\begin{array}{l}
l \\
i
\end{array}\right) S_{1}(n-l, m) \mathbb{C}_{i}^{(s)} C P_{l-i}^{(k)}(s ; \lambda, \mu)\right\} B_{n}^{(s)}(x) .
$$

Remark By the same method as Theorem 10, we have

$$
\begin{aligned}
& \hat{C} P_{n}^{(k)}(x ; \lambda, \mu) \\
& \quad=\sum_{m=0}^{n}\left\{\sum_{l=0}^{n-m} \sum_{i=0}^{l}\left(\begin{array}{l}
n \\
l
\end{array}\right)\left(\begin{array}{l}
l \\
i
\end{array}\right) S_{1}(n-l, m) \hat{\mathbb{C}}_{i}^{(s)} \hat{C} P_{l-i}^{(k)}(s ; \lambda, \mu)\right\} B_{m}^{(s)}(x) .
\end{aligned}
$$

For $C P_{n}^{(k)}(x ; \lambda, \mu) \sim\left(\left(1+e^{-\lambda t}\right)^{\mu} \frac{1}{\operatorname{Lif}_{k}(-t)}, e^{-t}-1\right), H_{n}^{(s)}(x \mid \lambda) \sim\left(\left(\frac{e^{t}-\lambda}{1-\lambda}\right)^{s}, t\right), s \in \mathbb{Z}_{\geq 0}, \lambda \in \mathbb{C}$ with $\lambda \neq 1$, let us assume that

$$
C P_{n}^{(k)}(x ; \lambda, \mu)=\sum_{m=0}^{n} C_{n, m} H_{m}^{(s)}(x ; \lambda)
$$

From (26), we have

$$
\begin{aligned}
C_{n, m}= & \frac{(-1)^{m}}{m !}\left\langle\left(1+(1+t)^{\lambda}\right)^{-\mu} \operatorname{Lif}_{k}(\log (1+t))\right. \\
& \times(1+t)^{-s}\left(1+\frac{\lambda}{\lambda-1} t\right)^{s}\left|(\log (1+t))^{m} x^{n}\right\rangle \\
= & \frac{(-1)^{m}}{m !} \sum_{l=0}^{n-m} m !\left(\begin{array}{c}
n \\
l+m
\end{array}\right) S_{1}(l+m, m) \sum_{i=0}^{\min \{s, n-l-m\}}\left(\begin{array}{l}
s \\
i
\end{array}\right)\left(\frac{\lambda}{\lambda-1}\right)^{i} \\
& \times\left\langle\left(1+(1+t)^{\lambda}\right)^{-\mu} \operatorname{Lif}_{k}(\log (1+t))(1+t)^{-s} \mid t^{i} x^{n-l-m}\right\rangle \\
= & (-1)^{m} \sum_{l=0}^{n-m} \sum_{i=0}^{\min \{s, n-l-m\}}\left(\begin{array}{c}
n \\
l+m
\end{array}\right)\left(\begin{array}{l}
s \\
i
\end{array}\right) \\
& \times(n-l-m)_{i}\left(\frac{\lambda}{\lambda-1}\right)^{i} S_{1}(l+m, m) C P_{n-l-m-i}^{(k)}(s ; \lambda, \mu) \\
= & (-1)^{m} \sum_{l=0}^{n-m} \sum_{i=0}^{\min \{s, l\}}\left(\begin{array}{c}
n \\
l
\end{array}\right)\left(\begin{array}{l}
s \\
i
\end{array}\right)(l)_{i}\left(\frac{\lambda}{\lambda-1}\right)^{i} S_{1}(n-l, m) C P_{l-i}^{(k)}(s ; \lambda, \mu) .
\end{aligned}
$$

Therefore, by (75) and (76), we obtain the following theorem.

Theorem 11 For $\lambda \in \mathbb{C}$ with $\lambda \neq 1, n \geq 0$, we have

$$
\begin{aligned}
& C P_{n}^{(k)}(x ; \lambda, \mu) \\
& =\sum_{m=0}^{n}(-1)^{m}\left\{\sum_{l=0}^{n-m} \sum_{i=0}^{\min \{s, l\}}\left(\begin{array}{l}
n \\
l
\end{array}\right)\left(\begin{array}{l}
s \\
i
\end{array}\right)(l)_{i}\right. \\
& \left.\quad \cdot\left(\frac{\lambda}{\lambda-1}\right)^{i} S_{1}(n-l, m) C P_{l-i}^{(k)}(s ; \lambda, \mu)\right\} H_{m}^{(s)}(x ; \lambda) .
\end{aligned}
$$


Remark By the same method as Theorem 11, we get

$$
\begin{aligned}
\hat{C} P_{n}^{(k)}(x ; \lambda, \mu) & \\
= & \sum_{m=0}^{n}\left(\sum_{l=0}^{n-m} \sum_{i=0}^{\min \{s, l\}}\left(\begin{array}{l}
n \\
l
\end{array}\right)\left(\begin{array}{l}
s \\
i
\end{array}\right)(l)_{i}\right. \\
& \left.\cdot\left(\frac{1}{1-\lambda}\right)^{i} S_{1}(n-l, m) \hat{C} P_{l-i}^{(k)}(0 ; \lambda, \mu)\right\} H_{m}^{(s)}(x ; \lambda) .
\end{aligned}
$$

For $C P_{n}^{(k)}(x ; \lambda, \mu) \sim\left(\left(1+e^{-\lambda t}\right)^{\mu} \frac{1}{\operatorname{Lif}_{k}(-t)}, e^{-t}-1\right)$ and $x^{(n)} \sim\left(1,1-e^{-t}\right)$, let us assume that

$$
C P_{n}^{(k)}(x ; \lambda, \mu)=\sum_{m=0}^{\infty} C_{n, m} x^{(m)}
$$

By (26), we get

$$
\begin{aligned}
C_{n, m} & =\frac{1}{m !}\left\langle\frac{1}{\left(1+e^{\lambda \log (1+t))^{\mu}}\right.} \operatorname{Lif}_{k}(\log (1+t))\left(1-e^{\log (1+t)}\right)^{m} \mid x^{n}\right\rangle \\
& =\frac{1}{m !}\left\langle\left(1+(1+t)^{\lambda}\right)^{-\mu} \operatorname{Lif}_{k}(\log (1+t))(-t)^{m} \mid x^{n}\right\rangle \\
& =\frac{(-1)^{m}}{m !}\left\langle\left(1+(1+t)^{\lambda}\right)^{-\mu} \operatorname{Lif}_{k}(\log (1+t)) \mid t^{m} x^{n}\right\rangle \\
& =(-1)^{m}\left(\begin{array}{c}
n \\
m
\end{array}\right)\left\langle\left(1+(1+t)^{\lambda}\right)^{-\mu} \operatorname{Lif}_{k}(\log (1+t)) \mid x^{n-m}\right\rangle \\
& =(-1)^{m}\left(\begin{array}{c}
n \\
m
\end{array}\right) C P_{n-m}^{(k)}(0 ; \lambda, \mu) .
\end{aligned}
$$

Therefore, by (77) and (78), we obtain the following theorem.

Theorem 12 For $n \geq 0$, we have

$$
C P_{n}^{(k)}(x ; \lambda, \mu)=\sum_{m=0}^{n}(-1)^{m}\left(\begin{array}{c}
n \\
m
\end{array}\right) C P_{n-m}^{(k)}(0 ; \lambda, \mu) x^{(m)} .
$$

Remark By the same method as Theorem 12, we get

$$
\hat{C} P_{n}^{(k)}(x ; \lambda, \mu)=\sum_{m=0}^{n}\left(\begin{array}{c}
n \\
m
\end{array}\right) \hat{C} P_{n-m}^{(k)}(0 ; \lambda, \mu)(x)_{m} .
$$

\section{Competing interests}

The authors declare that they have no competing interests.

\section{Authors' contributions}

All authors contributed equally to the manuscript and typed, read, and approved the final manuscript.

\section{Author details}

'Department of Mathematics, Sogang University, Seoul, 121-742, Republic of Korea. ${ }^{2}$ Department of Mathematics, Kwangwoon University, Seoul, 139-701, Republic of Korea. 


\section{Acknowledgements}

This work was supported by Kwangwoon University 2013.

Received: 12 October 2013 Accepted: 10 December 2013 Published: 06 Jan 2014

\section{References}

1. Ozden, H, Cangul, IN, Simsek, Y: Remarks on q-Bernoulli numbers associated with Daehee numbers. Adv. Stud. Contemp. Math. 18(1), 41-48 (2009)

2. Kim, T, Kim, DS, Mansour, T, Rim, S-H, Schork, M: Umbral calculus and Sheffer sequences of polynomials. J. Math. Phys. $54,083504(2013)$

3. Kim, T: Some identities on the $q$-Euler polynomials of higher order and $q$-Stirling numbers by the fermonic $p$-adic integral on $\mathbb{Z}_{p}$. Russ. J. Math. Phys. 16(4), 484-491 (2009)

4. Kim, DS, Kim, T, Lee, S-H: Poly-Cauchy numbers and polynomials with umbral calculus viewpoint. Int. J. Math. Anal. 7(45), 2235-2253 (2013)

5. Can, M, Cenkci, M, Kurt, V, Simsek, Y: Twisted Dedekind type sums associated with Barnes' type multiple Frobenius-Euler L-functions. Adv. Stud. Contemp. Math. 18(2), 135-160 (2009)

6. Carlitz, L: A note on Bernoulli and Euler polynomials of the second kind. Scr. Math. 25, 323-330 (1961)

7. Comtet, L: Advanced Combinatorics. Reidel, Dordrecht (1974)

8. Dere, R, Simsek, Y: Applications of umbral algebra to some special polynomials. Adv. Stud. Contemp. Math. 22(3), 433-438 (2012)

9. Gould, HW: Explicit formulas for Bernoulli numbers. Am. Math. Mon. 79, 44-51 (1972)

10. Kim, DS, Kim, T: Some identities of Bernoulli and Euler polynomials arising from umbral calculus. Adv. Stud. Contemp. Math. 23(1), 159-171 (2013)

11. Kim, DS, Kim, T, Kim, YH, Dolgy, DV: A note on Eulerian polynomials associated with Bernoulli and Euler numbers and polynomials. Adv. Stud. Contemp. Math. 22(3), 379-389 (2012)

12. Kim, DS, Kim, T, Dolgy, DV, Rim, SH: Some new identities of Bernoulli, Euler and Hermite polynomials arising from umbral calculus. Adv. Differ. Equ. 2013, 73 (2013)

13. Komatsu, T: On poly-Cauchy numbers and polynomials. Available at http://carma.newcastle.edu.au/alfcon/pdfs/Takao_Komatsu-alfcon.pdf

14. Merlini, D, Sprugnoli, R, Verri, MC: The Cauchy numbers. Discrete Math. 306, 1906-1920 (2006)

15. Roman, S: The Umbral Calculus. Pure and Applied Mathematics, vol. 111. Academic Press, New York (1984) ISBN:0-12-594380-6 x+193 pp.

10.1186/1687-1847-2014-4

Cite this article as: Kim and Kim: Poly-Cauchy and Peters mixed-type polynomials. Advances in Difference Equations $2014,2014: 4$

\section{Submit your manuscript to a SpringerOpen ${ }^{\ominus}$ journal and benefit from:}

- Convenient online submission

Rigorous peer review

- Immediate publication on acceptance

Open access: articles freely available online

- High visibility within the field

- Retaining the copyright to your article 